\title{
Automatic detection of multiple oriented blood vessels in retinal images
}

\author{
P. C. Siddalingaswamy ${ }^{1}$, K. Gopalakrishna Prabhu ${ }^{2}$ \\ ${ }^{1}$ Department of Computer Science \& Engineering, Manipal Institute of Technology, Manipal, India; \\ ${ }^{2}$ Department of Biomedical Engineering, Manipal Institute of Technology, Manipal, India. \\ Email: pcs.swamy@manipal.edu
}

Received 9 September 2009; revised 16 October 2009; accepted 19 October 2009.

\begin{abstract}
Automatic segmentation of the vasculature in retinal images is important in the detection of diabetic retinopathy that affects the morphology of the blood vessel tree. In this paper, a hybrid method for efficient segmentation of multiple oriented blood vessels in colour retinal images is proposed. Initially, the appearance of the blood vessels are enhanced and background noise is suppressed with the set of real component of a complex Gabor filters. Then the vessel pixels are detected in the vessel enhanced image using entropic thresholding based on gray level co-occurrence matrix as it takes into account the spatial distribution of gray levels and preserving the spatial structures. The performance of the method is illustrated on two sets of retinal images from publicly available DRIVE (Digital Retinal Images for Vessel Extraction) and Hoover's databases. For DRIVE database, the blood vessels are detected with sensitivity of $86.47 \pm 3.6$ $(M e a n \pm$ SD) and specificity of 96 \pm 1.01 .
\end{abstract}

Keywords: Blood Vessel Segmentation; Gabor Filter; Co-Occurence Matrix; Diabetic Retinopathy.

\section{INTRODUCTION}

In clinical ophthalmology colour retinal images acquired from digital fundus camera are widely used for detection and diagnosis of diseases like diabetic retinopathy, hypertension and various vascular disorders. Retinal images provide information about the blood supply system of the retina. Diabetic retinopathy is a disorder of the retinal vasculature that eventually develops to some degree in nearly all patients with long-standing diabetes mellitus [1]. The timely diagnosis and referral for management of diabetic retinopathy can prevent $98 \%$ of severe visual loss, for that, the patient has to undergo regular screening of eye for retinopathy. The process involves dilating the eyes with mydriatic drops and capturing the retinal image using standard digital colour fundus camera. Screening program results in large number of retinal images needed to be examined by ophthalmologists. Manual diagnosis is usually performed by analyzing the images from a patient, as not all images show signs of diabetic retinopathy, it increases the time and decreases the efficiency of ophthalmologists. Therefore, an automatic segmentation of the vasculature could save workload of the ophthalmologists and may assist in characterizing the detected lesions and to identify false positives [2]. Another important application of automatic retinal vessel segmentation is in the registration of retinal images of the same patient taken over period of time [3]. The registered images are useful in monitoring the progression of disease and to observe the effect of treatment.

Different techniques of segmentation of retinal images have been investigated so far. They are filter based methods, vessel tracking methods, classifier based methods and morphological methods. The techniques utilize the prior knowledge such as, contrast that exists between the blood vessels and surrounding background, origin of vasculature from the same point that is the optic disc and connectivity of the vessels. Filter based methods $[4,5]$ and [6] employ a two dimensional linear structural element that has a Gaussian cross-profile section to identify the blood vessels, which typically has a Gaussian profile. The gaussian kernel is rotated into different orientations to fit into vessels of different configuration to obtain a vessel enhanced image. The image is then thresholded to extract the vessel part from the background. In Hoover et al., 2000 [4] threshold is computed using piece wise threshold probing of matched filter response image. This works well on images of healthy retina, but in diseased states such as diabetic retinopathy it results in detection of many false positives. These methods suffer from problems associated with detecting smaller and tortuous vessels that are prone to changes in background intensity. Vessel tracking methods [7] and [8] use a model to track the vessels starting at given points. Here each vessel segment is defined by three attributes which are direction, width, and center point. Individual segments are identified using a search procedure which keeps track of the center of the vessel and makes some decisions about the future 


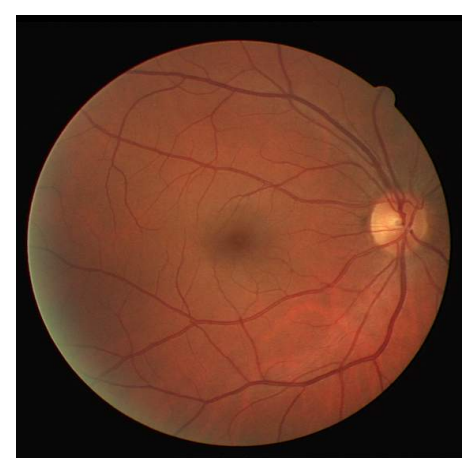

(a)

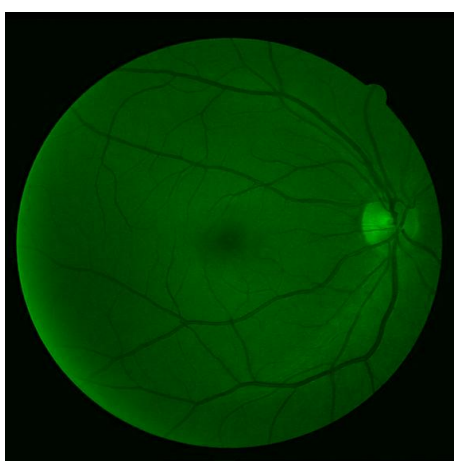

(c)

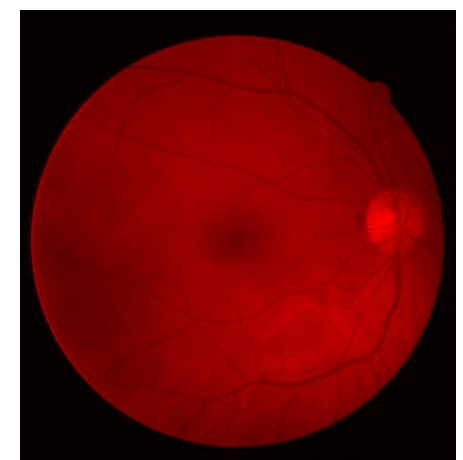

(b)

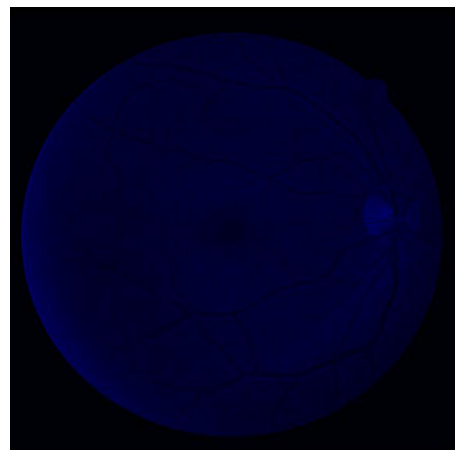

(d)

Figure 1. (a) Colour retinal image; (b-d) Red, Green and Blue component images.

path of the vessel based on certain vessel properties. These methods require that beginning and ending search points are manually selected using cursor or by using simple thresholding techniques. Vessel tracking methods provide very accurate measurements of vessel widths but tracking methods often tend to terminate at branch points. Classifier-based method employs two-step approach [9]. They start with a segmentation step often by employing one of the mentioned matched filter-based methods and then the regions are classified according to many features. In the next step neural networks classifier is constructed using selected features by the sequential forward selection method with the training data to detect vessel pixels. Mathematical Morphology is employed for segmentation of blood vessels as reported in $[10,11]$ and [12]. These methods exploits features of the vasculature shape that are known prior, such as it being piecewise linear and connected. They work well on normal retinal images with uniform contrast but suffer when there is a noise due to pathologies within the retina of eye. Many papers have reported work on segmentation of vessels, but still there is scope for improvement as these methods detect vessels along with artifacts. Also detection process becomes much more complicated in presence of lesions and other pathological changes affect the retinal images.

The proposed retinal vessel detection method is comprised of two steps that is the retinal vessel enhancement followed by entropic thresholding. A set of Gabor filters tuned to particular frequency and orientation are used to enhance the blood vessels suppressing the background. Entropy based thresholding based on gray level co-occurrence matrix is employed for the segmentation of the vessels. The following sections elucidate materials and methods for vessel segmentation method, results and discussion.

\section{MATERIALS AND METHODS}

To develop a retinal vessel segmentation system, the first important thing is to obtain an effective database. To realize this and also for facilitating comparison with the existing methods, two sets of publicly available databases are used.

\subsection{Retinal Image Database}

The DRIVE database provides forty images [19]. The images are acquired using a Canon CR5 non-mydriatic 3CCD camera with a 45 degree field of view (FOV). Each image was captured using 8 bits per colour plane at $768 \times 584$ pixels. The FOV of each image is circular with a diameter of approximately 540 pixels. The Hoover's database [4] consists of twenty digitized slides captured by a TopCon TRV-50 fundus camera at 35 FOV. The slides were digitized to $700 \times 605$ pixels with eight bits per colour channel. Both the databases provide the hand labeled blood vessels identified by experts as gold standard and binary mask image identifying the boundary of the effective portion of each image. Apart from these 


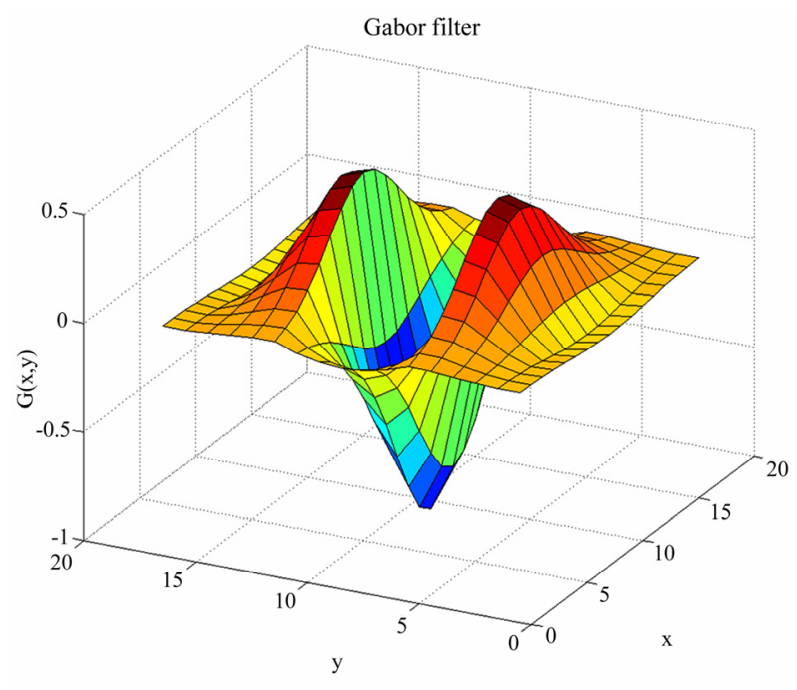

(a)

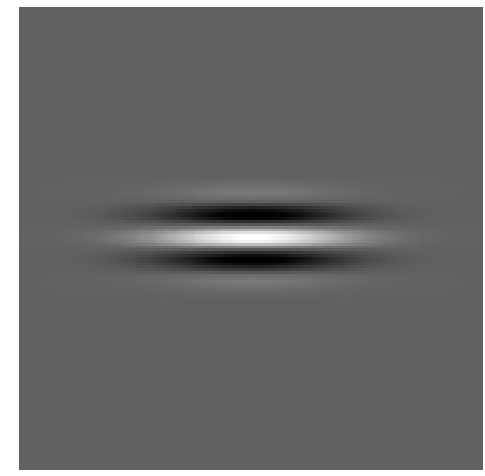

(b)

Figure 2. (a) Surface representation of Gabor filter; (b) Real part of Gabor filter.

two databases the images are also acquired from the Department of Ophthalmology, Kasturba medical college (KMC), Manipal using Sony FF450IR digital colour fundus camera with 24 bit colour depth and $768 \times 576$ pixel resolution. These images are of very large variability in terms of fundus disease and image quality and were used to test the robustness of proposed retinal vessel detection method.

\subsection{Preprocessing}

In the colour retinal images, blood vessels appear darker than the background similar to the colour of lesions like microaneurysms and hemorrhages. So it becomes essential to exempt the vessel area during the detection of lesions to avoid false positives. Only one step is involved in the preprocessing of retinal images for segmentation of vessels. It can be seen in the Figure 1 that the blood vessels appear most contrasted in the green channel compared to red and blue channels in RGB image. Only the green channel image is used for further processing suppressing the other two colour components.

\subsection{Vessel Enhancement}

The Gabor filters are widely applied to image processing and computer vision application problems such as face recognition and texture segmentation, strokes in character recognition and roads in satellite image analysis $[13,14]$ and $[15]$. Since, the vessels in the retinal image are connected and piecewise linear, for their segmentation gabor filters are better suited as they are capable of detecting oriented features and can be fine tuned to specific frequencies. Because of their frequency sensitiveness it is possible to filter out the background noise of retinal images. The spatial Gabor filter kernels are sinusoids modulated by a Gaussian window, the real part of which is expressed by

$$
g(x, y)=\exp \left[-\pi\left(\frac{x_{p}^{2}}{\sigma_{x}}+\frac{y_{p}^{2}}{\sigma_{y}}\right)\right] \cos \left(2 \pi f x_{p}\right)
$$

where,

$$
\begin{gathered}
x_{p}=x \cos \theta+y \sin \theta \\
y_{p}=-x \sin \theta+y \cos \theta
\end{gathered}
$$

The Gabor function is defined by five parameters as follows. $\theta$ is the orientation of the filter, an angle of zero gives a filter that responds to vertical features. $f$ is the central frequency of pass band. $\sigma_{x}$ is the standard deviation of Gaussian in $\mathrm{x}$ direction along the filter that determines the bandwidth of the filter. $\sigma_{y}$ is the standard deviation of Gaussian across the filter that control the orientation selectivity of the filter. The parameters are to be derived by taking into account the size of the lines or curvilinear structures to be detected. In retinal images the width of the vessels varies along the length of the vessel and in manual segmentation it is found that majority of the vessel diameters are of 4 pixels wide. Therefore to accommodate the all vessels in the detection the thickness parameter $t$ is set to four. The other parameters are derived using the procedure in [15] as $f=0.5 / t, \sigma_{x}=\lambda t / 0.75 \pi$ and $\sigma_{y}=0.85 \sigma_{x}$ and $\lambda=\sqrt{2 \ln 2 / \pi}$.

The surface representation and real part of resulting Gabor kernel is shown in Figure 2. It can be seen that it is suited for the orientation of directional features to provide good response for pixels associated with retinal blood vessels.

To obtain good response of the vessels oriented along 


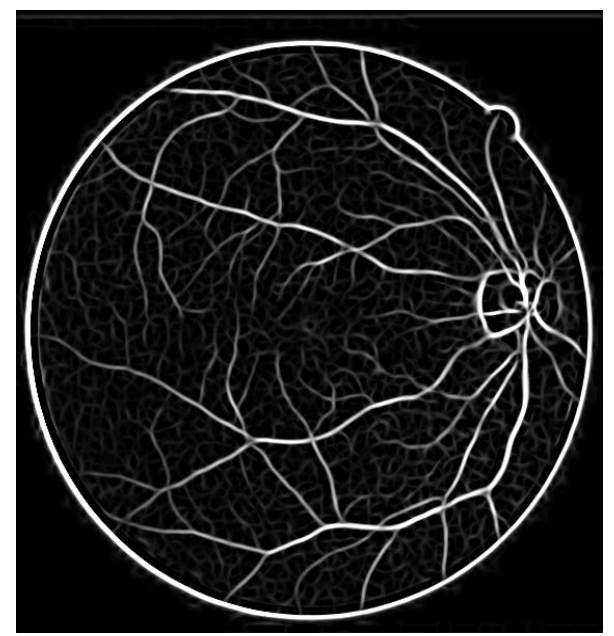

Figure 3. Enhanced vessels in Gabor response.
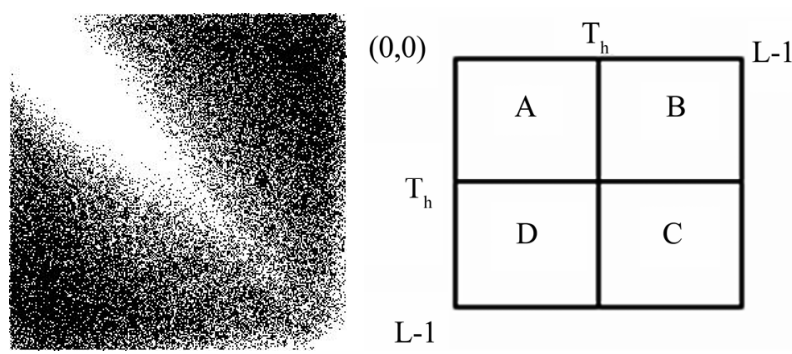

L-1

Figure 4. (a) Gray level distribution in GLCM; (b) Representation of GLCM of 4 quadrants

different directions, the $\theta$ of filter is rotated from $0^{\circ}$ to $170^{\circ}$ in the steps of ten degrees to produce a single peak response on the center of a vessel segment. At each pixel only the maximum response is retained. Figure 3 shows the result of convolving the image in Figure 1(c) with the set of gabor filters. It can be seen that the vessels are enhanced and background is suppressed considerably.

To obtain good response of the vessels oriented along different directions, the $\theta$ of filter is rotated from $0^{\circ}$ to $170^{\circ}$ in the steps of ten degrees to produce a single peak response on the center of a vessel segment. At each pixel only the maximum response is retained. Figure 3 shows the result of convolving the image in Figure 1(c) with the set of gabor filters. It can be seen that the vessels are enhanced and background is suppressed considerably.

\subsection{Entropic Thresholding}

Thresholding is used to segment the blood vessels from the background. An entropy based thresholding method based on gray level co-occurrence matrix (GLCM) is used to find optimal threshold as it takes into account the spatial distribution of gray levels and preserves the spatial structures in thresholded image [16,17] and [18].
Gray level co-occurrence matrix contains information on the distribution of gray level frequency and edge information, as it is very useful in finding the threshold value.

The gray level co-occurrence matrix $T=\left[t_{i, j}\right]$ of the image $I$ with $M \times N$ dimensional matrix gives an idea about the transition of intensities between adjacent pixels, indicating spatial structural information of an image. Depending upon the ways in which the gray level $i$ follows gray level $j$, different definitions of co-occurrence matrix are possible. The GLCM is obtained as follows:

$$
t_{i, j}=\sum_{l=1}^{M} \sum_{k=1}^{N} \delta
$$

where,

$$
\delta=1 \quad \text { if }\left\{\begin{array}{r}
f(l, k)=i \text { and } f(l, k+1)=j \\
f(l, k)=i \text { and } f(l+1, k)=j
\end{array}\right.
$$

$$
\delta=0 \quad \text { otherwise }
$$

The probability of co-occurrence $P_{i j}$ of gray levels $i$ and $j$ is written as

$$
P_{i, j}=\frac{t_{i, j}}{\sum_{i} \sum_{j} t_{i, j}}
$$

Let $T_{h}$ be the threshold within the range $\mathrm{O}_{\mathrm{h}} T_{h} L-1$, where $L$ is the number of gray levels. Threshold $T_{h}$ partitions the GLCM into four quadrants, namely A, B, C, and $\mathrm{D}$ as in Figure 4. Quadrant A represents gray level transition within the object while quadrant $\mathrm{D}$ represents gray level transition within the background. The gray level transition between the object and the background or across the object's boundary is placed in quadrant B and quadrant $\mathrm{C}$.

The probabilities of object class and background class are defined as

$$
P_{A}=\sum_{i=0}^{T_{h}} \sum_{j=0}^{T_{h}} P_{i, j}
$$

Table 1. Performance of retinal blood vessels segmentation method.

\begin{tabular}{cccc}
\hline Database & $\begin{array}{c}\text { No. of } \\
\text { images }\end{array}$ & $\begin{array}{c}\text { Sensitivity } \\
(\%)\end{array}$ & $\begin{array}{c}\text { Specificity } \\
(\%)\end{array}$ \\
\hline DRIVE & 40 & $86.47 \pm 3.6$ & $96 \pm 1.01$ \\
\hline
\end{tabular}

Table 2. Comparison of vessel segmentation results on Hoover's database.

\begin{tabular}{ccc}
\hline Method & $\begin{array}{c}\text { Sensitivity range } \\
(\%)\end{array}$ & $\begin{array}{c}\text { Specificity range } \\
(\%)\end{array}$ \\
\hline Proposed method & $79-91$ & $94-98$ \\
Hoover et al.2000 & $80-90$ & $92-93$ \\
\hline
\end{tabular}




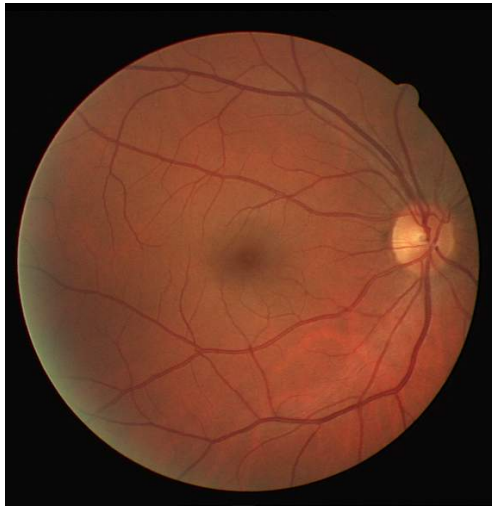

(a)

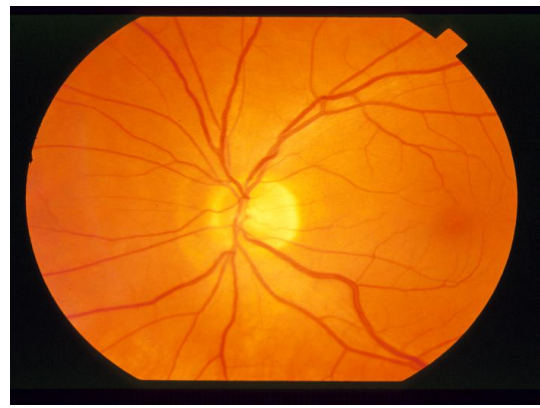

(d)

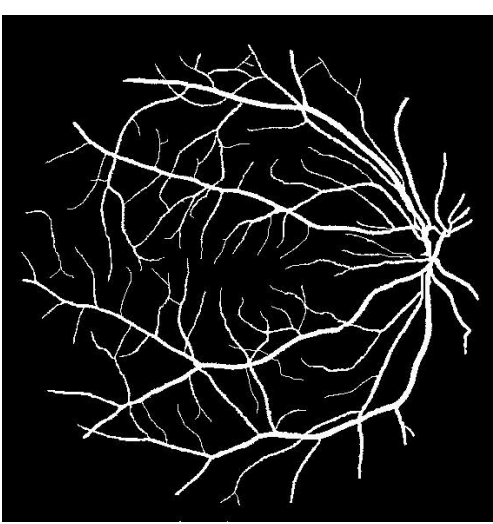

(b)

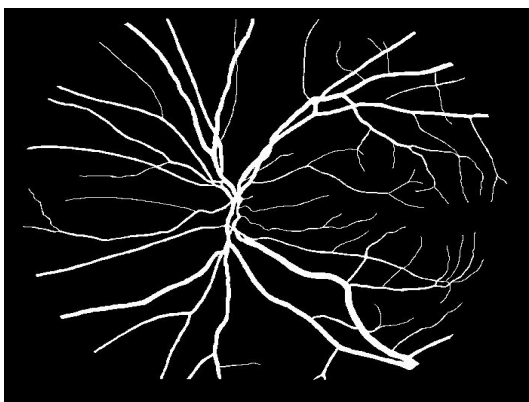

(e)

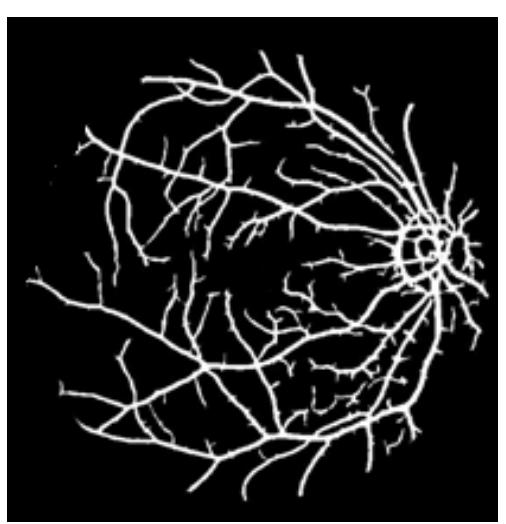

(c)

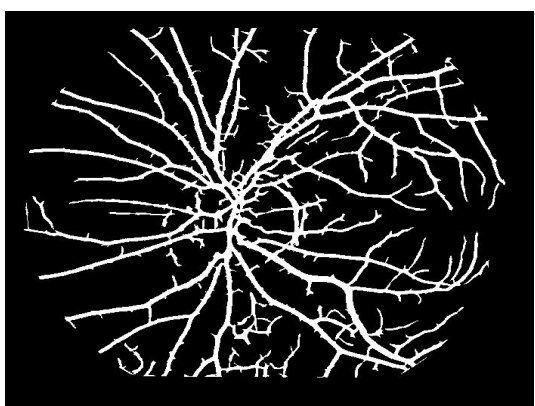

(f)

Figure 5. Retinal vessel segmentation. (a) Image from DRIVE database; (b) Corresponding manual segmentation; (c) Vessel segmentation result; (d) Image from Hoover's database; (g) Corresponding manual segmentation; (f) Vessel segmentation result.

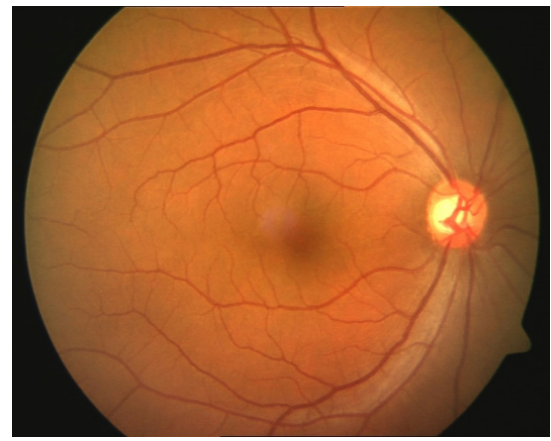

(a)

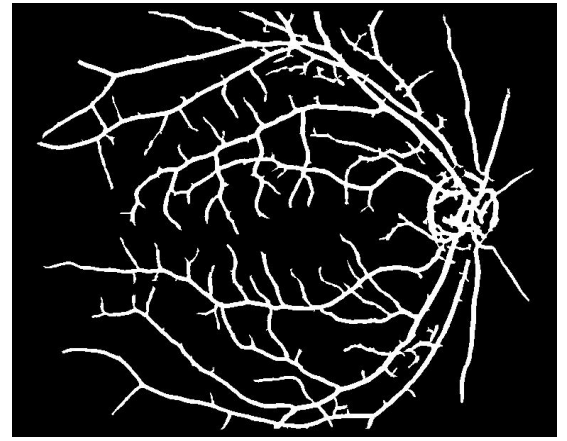

(b)

Figure 6. (a) Digital color retinal image from KMC database; (b) Segmented vessels.

$$
P_{C}=\sum_{i=T_{h}+1}^{L_{h 1}} \sum_{j=T_{h}+1}^{L_{h 1}} P_{i, j}
$$

Using (4) and (5) as normalization factors, the normalized probabilities of the object class and background class are functions of threshold vector $\left(T_{h}, T_{h}\right)$ are defined as

$$
\begin{gathered}
P_{i, j}^{A}=\frac{P_{i, j}}{P_{A}} \\
P_{i, j}^{C}=\frac{P_{I, J}}{p_{c}}
\end{gathered}
$$

The second-order entropy of the object is given by

$$
H_{A}\left(T_{h}\right)=h \frac{1}{2} \sum_{i=0}^{T_{h}} \sum_{j=0}^{T_{h}} P_{i, j}^{A} \log _{2} P_{i, j}^{A}
$$

Similarly, the second-order entropy of the background is given by

$$
H_{C}\left(T_{h}\right)=h \frac{1}{2} \sum_{i=T_{h}+1}^{L_{h 1}} \sum_{j=T_{h}+1}^{L_{h 1}} P_{i, j}^{C} \log _{2} P_{i, j}^{C}
$$

The total second-order local entropy of the object and the background is given by 


$$
H_{T}\left(T_{h}\right)=H_{A}\left(T_{h}\right)+H_{C}\left(T_{h}\right)
$$

Finally $T_{E}$ the gray level corresponding to the maximum of $H_{T}\left(T_{h}\right)$ gives the optimal threshold for vessel and non vessel classification.

$$
T_{E}=\arg \left[\max _{T=0}^{\max } H_{h}\left(T_{h}\right)\right]
$$

\section{RESULTS AND DISCUSSION}

Retinal images from the DRIVE database and Hoover's database are used for the automatic segmentation of retinal vessels. Figure 5 shows the manual segmented image and the final output obtained by the proposed method on the images from both the image databases. On Windows XP, Intel PC with $1.66 \mathrm{GHz} \mathrm{CPU}$ and $512 \mathrm{MB}$ memory using Matlab 7.0, the method takes about 20 seconds to detect the vessels in retinal image.

The performance of the method is evaluated using sensitivity and specificity at pixel level in comparison with manually segmented vessels by an expert.. Sensitivity gives the percentage of pixels correctly classified as vessels by the method and specificity gives the percentage of non vessels pixels classified as non vessels by the method. given by

$$
\begin{gathered}
\text { Sensitivity }=\frac{T_{p}}{T_{p}+F_{n}} \\
\text { Specificity }=\frac{T_{n}}{T_{n}+F_{P}}
\end{gathered}
$$

where $T_{p}$ is true positive, $T_{n}$ is true negative, $F_{p}$ is false positive and $F_{n}$ is false negative at each pixel. Table 1 show the performance of the proposed method on forty images from DRIVE database.

The results of the proposed method are also compared with those of Hoover et al. 2000 [4] on twenty images from the Hoover's database. The database has ten normal and ten abnormal retinal images and the result is depicted in Table 2. It can be seen that the proposed method performs better with lower specificity even in the presence of lesions in the abnormal images.

Apart from two standard databases the method is also tested on retinal images obtained from Ophthalmology department, KMC. These images are of large variability in terms of presence of lesions and image quality. These are considered to evaluate the robustness of the method. The result for one of the image is shown in Figure 6 and is validated by ophthalmologists.

\section{CONCLUSIONS}

An efficient method for automatic segmentation of retinal blood vessels has been presented. Images from three different datasets are used to evaluate the robustness and accuracy of the method, demonstrating that it may be useful in a wide range of retinal images. Based on a brief comparison with some other vessel segmentation algorithms, we can conclude that the Gabor filter and entropic threshold provides a better segmentation. The segmented vessels can be used to obtain the control points used in the retinal registration techniques. It is hoped that automated segmentation of vessel technique can detect the signs of diabetic retinopathy in the early stage, monitor the progression of disease, minimize the examination time and assist the ophthalmologist for a better treatment plan.

\section{ACKNOWLEDGEMENT}

Our sincere thanks to ophthalmologists of the Department of Ophthalmology, Kasturba Medical College, Manipal for providing the necessary images and clinical details.

\section{REFERENCES}

[1] Emily, Y.C. (2003) Diabetic retinopathy. Preferred Practice Patterns, American Academy of Ophthalmology, USA.

[2] Thomas, W., Klein, J.C., Pascale, M. and Ali, E. (2002) A contribution of image processing to the diagnosis of diabetic retinopathy-detection of exudates in color fundus images of the human retina. IEEE Trans. Medical. Imaging, 21, 1236-1243.

[3] Laliberté, F., Gagnon, L. and Sheng, Y. (2003) Registration and fusion of retinal images: an evaluation study. IEEE Trans. Medical. Imaging, 22, 661-673.

[4] Hoover, A., Kouznetsoza, V., Goldbaum, M. (2000) Locating blood vessels in retinal images by piecewise threshold probing of a matched filter response. IEEE Transactions on Medical Imaging, 19, 203-210.

[5] Chaudhuri, S., Chatterjee, S., Katz, N., Nelson, M. and Goldbaum, M. (1989) Detection of blood vessels in retinal images using two dimensional matched filters. IEEE transactions on Medical Imaging, 8, 263-269.

[6] Thitiporn, C. and Fan, G.L. (2003) An efficient Algorithm for extraction of anatomical structures in retinal images. Proc. Of Intl. Conf. on Image Processing, 1, 1093-1096.

[7] Wu, D., Zhang, M., Liu, J.C. and Wendall, B. (2006) On the adaptive detection of blood vessels in retinal images. IEEE Transactions on Biomedical Engineering, 53, 341-343.

[8] Pinz, A., Bernogger, S., Datlinger, P. and Kruger, A. (1998) Mapping the human retina. IEEE Transactions on Medical imaging, 17, 606-619.

[9] Sinthanayothin, C., Boyce, J.F., Cook, H.L. and Williamson H.T. (1999) Automated location of the optic disc, fovea, and retinal blood vessels from digital color fundus images. British Journal of Ophthalmology, 83, 902-910.

[10] Zana, F. and Klein, J. (2001) Segmentation of vessel-like patterns using mathematical morphology and curvature evaluation. IEEE Transactions on Image Processing, 10, 1010-1019.

[11] Siddalingaswamy, P.C., Prabhu, G.K. and Mithun, D. (2006) Feature extraction of retinal image. Proc. Of the National Conference for $P G$ and Research scholars, 24-27. 
[12] Yang, Y., Huang, S.Y. and Rao, N. (2008) An automatic hybrid method for retinal blood vessel extraction. Journal of Appl. Math. Comp. Sci., 18, 399-407.

[13] Lee, T.S. (1996) Image representation using 2D Gabor wavelets. IEEE Transactions of Pattern Analysis and Machine Intelligence. 18, 959-971.

[14] Chen, J., Sato, Y. and Tamura, S. (2000) Orientation space filtering for multiple orientation line segmentation. IEEE Transactions of Pattern Analysis and Machine Intelligence, 22, 417-429.

[15] Liu, Z.Q., Cai, J. and Buse, R. (2003) Hand-writing recognition: soft computing and probablistic approaches. Springer Verlag. Berlin.

[16] Yang, C.W., Chung, P.C. and Chang, C.I. (1996) Hierar- chical fast two dimensional entropic thresholding algorithm using a histogram pyramid. Optical Engineering, 35, 3227-3241.

[17] Mark, L. Althouse, G. and Chang, C. (1995) Target detection in multispectral images using the spectral co- occurrence matrix and entropy thresholding. Optical Engineering, 34, 2135-2148.

[18] Mokji, M.M. and S.A.R and Bakar A. (2007) Adaptive thresholding based on co occurrence matrix edge information. Journal of Computers, 2, 44-52.

[19] Staal, J.J., Abràmoff, M.D., Niemeijer, M., Viergever, M. A. and van Ginneken, B. (2004) Ridge based vessel segmentation in color images of the retina. IEEE Trans. Med. Imag., 23, 501-509. 